\title{
Welche Binde bringt den besten Druck?
}

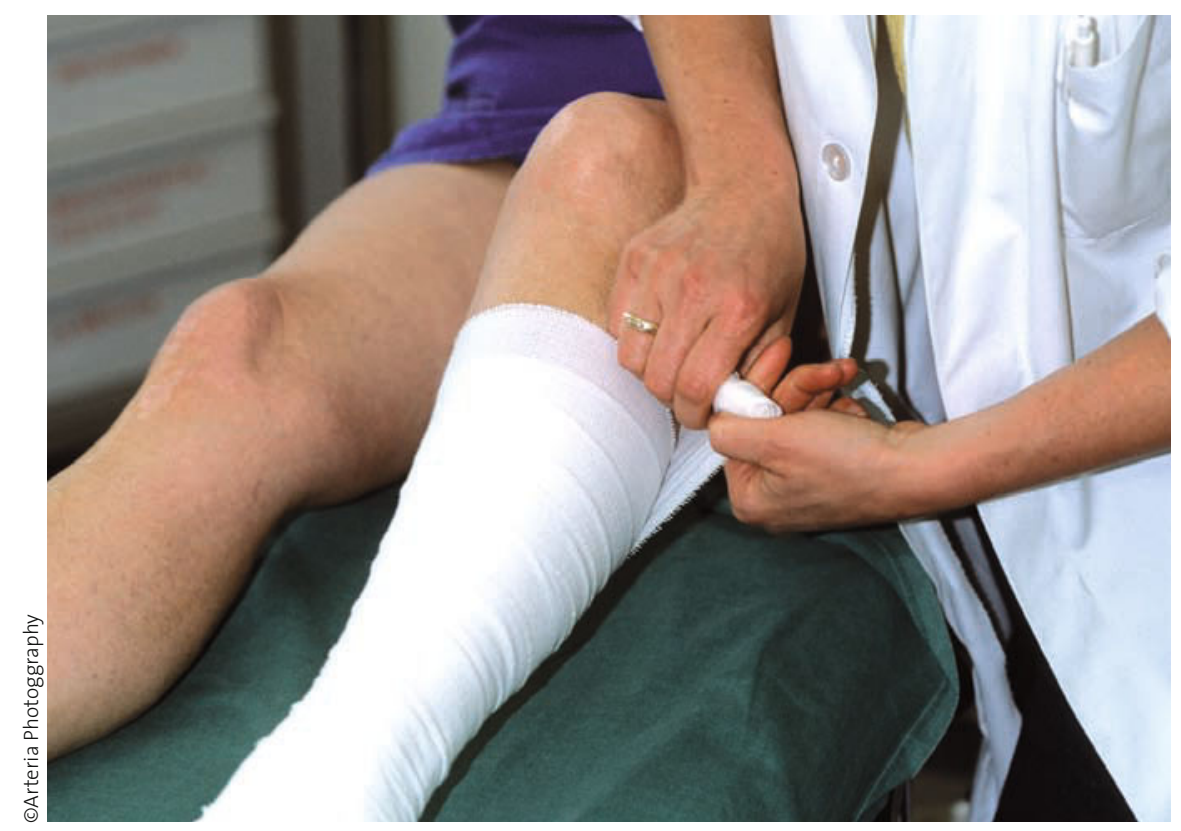

Neue Bindensysteme sollen die Kompressionsbehandlung wesentlich vereinfachen.

Zweilagige Kompressionsbinden mit kontrolliert applizierbarem Druck stellen ein Optimum zwischen Druck und Tragekomfort dar und sind Grundlage einer erfolgreichen Therapie des Ulcus cruris.

- Die Kompressionstherapie mit einem Druck zwischen 30 und $50 \mathrm{mmHg}$ über dem Knöchel ist der Goldstandard für die Behandlung für Ulcera curis. Allerdings ergeben sich bei der Therapie einige Probleme mit der langfristigen Erhaltung dieses Drucks durch eine entsprechende Kompressionsbinde, die korrekte Anlage der Binde und der Toleranz des Patienten. Eine zu geringe Kompression ist ineffektiv, $\mathrm{zu}$ hohe Kompressionsdrücke werden nicht toleriert und können sogar gefährlich sein.

Bei 24 gesunden Freiwilligen hat man nun mit einem technisch aufwendigen Druck-Registrierungssystem den Interface-Druck dreier Kompressionsbinden unmittelbar nach der Anlage sowie nach einem, nach drei und nach sieben Tagen bestimmt. Der druckaufnehmende Sensor war über dem Innenknöchel positioniert. Zusätzlich wurde das Volumen der unteren Extremität an den Tagen 0 und 7 mithilfe eines dreidimensionalen Imagesystems bestimmt und die Zufriedenheit der Probanden anhand eines Fragebogens evaluiert. Verglichen wurde der Effekt einer zweilagigen, einer vierlagigen und einer Kurzzug-Kompressionsbinde.

Die Daten von 36 der insgesamt 48 potenziell auswertbaren Beine konnten analysiert werden. Es zeigte sich, dass die Kurzzugbinde am dritten Tag einen signifikanten höheren Druckverlust aufwies als die zweilagigen und die vierlagigen Binden. Zwischen den beiden mehrlagigen Binden beobachtete man zu keinem Zeitpunkt einen signifikanten unterschiedlichen Druckverlust im Lauf der Studiendauer, sodass der erforderliche therapeutische Druck von $40 \mathrm{~mm}$ $\mathrm{Hg}$ über die ganze Studiendauer hinweg aufrechterhalten werden konnte.

Allerdings bestanden zwischen der zweilagigen und der vierlagigen Binde Unterschiede in der Toleranz und der Praktikabilität. Im Hinblick auf die Immobilisierung im Sprunggelenk, das Verrutschen und die Lockerung der Bandage, einen störenden Effekt beim
Schlaf, beim Sitzen und beim Gehen schnitt die zweilagige Binde signifikant besser $a b$ als die vierlagige. Beide mehrlagigen Binden führten zu einer signifikant höheren Volumenreduktion der unteren Extremität im Vergleich zur Kurzzugbinde.

\section{Kommentar}

Die Kompressionstherapie von Ödemen und Ulcera cruris ist eine sehr effektive Behandlung, allerdings nur, wenn sie technisch in der richtigen Weise und mit gutem Material ausgeführt wird. Zur Kontrolle des Bindenzugs ist es erforderlich, dass die Binde eine Hilfsmarkierung aufweist, z. B. aufgedruckte Ellipsen, die sich bei korrektem Zug zu Kreisen verformen. Kurzzugbinden sind zwar leichter anzubringen und belasten den Patienten weniger, lockern sich aber nach kurzer Zeit und beginnen zu verrutschen. Die zweilagigen Kompressionsbinden-Systeme stellen wahrscheinlich ein Optimum zwischen Effektivität und Praktikabilität dar. Allerdings ist es mit gutem Material alleine nicht getan; mindestens ebenso wichtig ist die korrekte Wickeltechnik.

H. S. FÜEßL =

- M. Jünger et al.

(juenger@uni-greifswald.de); Department of Dermatology, University Hospital of Greifswald, Greifswald, Germany. Comparison of interface pressures of three compression bandaging systems used on healthy volunteers. Journal of Wound Care, 18 (2009), 11, 474-480 